\title{
Attentional Control in The Rostromedial Prefrontal Cortex in a Robot- Assisted Task: an Fnirs Study
}

\section{Duc Trung Le}

Hiroshima University

Kazuki Watanabe

Hiroshima University

\section{Hiroki Ogawa}

Hiroshima University

\section{Kojiro Matsushita}

Gifu University

Naoki Imada

Araki Neurosurgical Hospital

\section{Shingo Taki}

Araki Neurosurgical Hospital

\section{Yuji Iwamoto}

Araki Neurosurgical Hospital

\section{Takeshi Imura}

Hiroshima Cosmopolitan University

\section{Hayato Araki}

Araki Neurosurgical Hospital

\section{Osamu Araki}

Araki Neurosurgical Hospital

\section{Taketoshi Ono}

University of Toyama

Hisao Nishijo

University of Toyama

Naoto Fujita

Hiroshima University

Susumu Urakawa ( $\square$ urakawas@hiroshima-u.ac.jp )

Hiroshima University

\section{Research Article}

Keywords: robot-assisted tasks, RES, NON, ROB, hemodynamic responses, rmPFC processes 
Posted Date: May 10th, 2021

DOl: https://doi.org/10.21203/rs.3.rs-505668/v1

License: (c) (i) This work is licensed under a Creative Commons Attribution 4.0 International License. Read Full License 


\section{Abstract}

During robot-assisted tasks, central nervous system may attend to external information-processing mode, termed as external attending. However, the neural mechanisms underlying this process remain unclear. The rostromedial prefrontal cortex (rmPFC) may be the core of an attentional control system that biases the allocation of attentional resources toward external information-processing when external attending is required for ongoing task performance. Here, we employed functional near-infrared spectroscopy to investigate hemodynamic responses in rmPFC subregions and additional frontal-parietal cortices during robot-assisted motor execution. Elbow flexion-extension movements, which require attention to kinematic consistency in range-of-motion, were employed as a motor task. Eighteen participants performed the task in three loading conditions requiring different degrees of external attending in ascending order: nonloading (NON), resistive loading (RES), and robotic assistive loading condition (ROB). Hemodynamic responses in the ventral and dorsal rmPFC were higher during ROB than during NON. Responses in the ventral rmPFC were significantly higher during ROB than during RES. Further, hemodynamic responses in the dorsal rmPFC during ROB were positively correlated with kinematic variability. These data suggest that robot-assisted motor execution involves rmPFC processes to bias the allocation of attentional resources toward external information-processing.

\section{Introduction}

Stroke is the second most common cause of mortality and the third most common cause of disability worldwide [1]. Despite considerable progress in the management of acute stroke, many stroke survivors experience various functional deficits, which diminish their ability to fulfill daily tasks. Robotic devices have emerged as a rehabilitation tool capable of providing task-specific, intensive, and multi-sensory training [2-4] while reducing manpower and labor involvement during the therapeutic process. Among multiple types of robots developed, assistive exoskeleton robots based on human motion intention are a promising technology given their ability to augment the performance and improve the motor function of users [5-8]. Determining the effects of robot-assisted rehabilitation requires a deeper understanding of the mechanisms underlying human-robot interactions.

When the human mind is occupied with one task, the central nervous system (CNS) involves relevant information-processing to execute the task. An important feature of the high-level executive function is cognitive processes that allocate attentional resources (central executive resources) to processing taskrelated information. This ability refers to processing modes by which brain networks attend to, which can be classified as external and internal attending. In particular, external attending involves the allocation of attentional resources to processing the external information perceived through senses $[9,10]$. In contrast, internal attending engages the attentional resource allocation to processing of internally generated information such as memory or mental imagery [10-12]. It has been suggested that attentional resources are limited in capacity [13]. Therefore, to perform or learn a given task, the subject involves a certain limited amount of this capacity, requiring brain networks to flexibly shift to external or internal attending, according to current task goals $[14,15]$. 
Multiple lines of evidence suggest that execution with an assistive exoskeleton robot requires the CNS to focus on external attending. For instance, robot-assisted motor learning is strongly associated with augmented somatosensory feedback processing by not only the subject's movements but also external assistance [16-18]. In particular, human-robot interactions provide robust somatosensory feedback of assisted movements to CNS in order to maintain motor performance consistently. Such feedback is considered an augmented indemnification for impairments in afferent sensory information, which facilitates the improved generation of internal dynamic models for movement guidance in the performer. These processes form closed-loop action-perception pathways that promote sensorimotor stimulation which drives brain plasticity. Therefore, the processing of external information plays a crucial role in motor learning with robotic assistance. Further, execution with an assistive exoskeleton robot requires performers to interact their voluntary movements with robotic assistance generated according to their own motion intentions. As such, somatosensory feedback provides valuable information for both feedforward and feedback control of human-robot interactions $[16,19]$. Thus, the external informationprocessing contributes substantially to motor performance and learning of robot-assisted execution. This necessitates prioritization of external attending during human-robot interactions. Although the involvement of the sensorimotor system in robot-assisted execution has been investigated extensively [20-24], the underlying neural mechanisms that govern the allocation of attentional resources during motor execution with an assistive exoskeleton robot have not been elucidated.

Considerable evidence implicates the rostral prefrontal cortex (PFC) as a crucial mediator of top-down control which drives the allocation of attentional resources. The rostral-most portion of the PFC, termed the frontopolar cortex corresponding to Brodmann area 10, is widely known for its involvement in diverse high-level cognitive functions [25-27]. The rostral PFC is anatomically interconnected with supramodal cortices in the PFC, anterior temporal cortex, and cingulate cortex [28-30]. These connections enable the rostral PFC to evaluate and modulate multimodal integration of processed information from downstream sensory systems. Furthermore, the rostral PFC has functional connections with subcortical regions including the thalamus and striatum [31]. This connectivity underpins the PFC's ability to modulate information-processing [32,33]. Based on these anatomical and functional connections, the rostral PFC forms the apex of hierarchical control organization [34,35] and mediates specific cognitive abilities by deploying top-down control in allocating attentional resources.

Burgess et al. have put forth a "gating hypothesis" [15] which proposes that the rostral PFC is engaged in a supervisory attentional gateway (SAG) system as a core region that generates attentional bias toward the attending mode which has a stronger influence on ongoing task performance. Specifically, the gating hypothesis suggests that the flow of information-processing continuously competes for attentional resources and requires minimal recruitment of the SAG system in well-specified or familiar conditions. SAG system involvement strongly influences the gain between attending modes when action selection via this competition is inadequate to produce optimal behavior. Burgess et al. further conjectured that functional organization occurs along the medial-lateral axis within the rostral PFC. Under this framework, the lateral part of the rostral PFC (rIPFC) biases toward internal attending, whereas the medial part of the rostral PFC (rmPFC), especially its most anterior subdivision, prioritizes external attending. In support of 
this hypothesis, converging neuroimaging evidence has revealed increased activation in the rmPFC during task performance requiring external attending [31,36,37]. Collectively, these findings suggest that the interaction between humans and assistive exoskeleton robots involves top-down rmPFC attentional control to bias the allocation of attentional resources toward external information-processing.

Nevertheless, the contribution of the rmPFC to robot-assisted execution has not been reported to date.

Functional near-infrared spectroscopy (fNIRS) is emerging as a practical imaging tool for assessing cortical activity of the cerebrum during motion-demanding tasks. fNIRS is a non-invasive and low-cost optical technique that measures concentration changes in oxygenated hemoglobin (oxy-Hb) and deoxygenated hemoglobin (deoxy-Hb) in cortical microvasculature. Mean cortical activation and alterations in regional cerebral blood flow are associated with an increase in oxy-Hb and a decrease in deoxy- $\mathrm{Hb}$ [38]. As this modality does not require the immobilization of participants, fNIRS has been applied in many motor control studies involving free upper limb movement [39], walking [40], and social interaction [41]. fNIRS is thus an optimal neuroimaging approach for investigating cortical activity of the cerebrum during human-robot interactions.

In the present study, we aimed to investigate cortical hemodynamic responses in rmPFC subregions and additional frontal-parietal cortices during motor execution with an assistive exoskeleton robot. We employed a single-joint version of the Hybrid Assistive Limb (HAL-SJ) system, which is a wearable exoskeleton robot developed to aid upper and lower limb functions [42]. HAL-SJ provides robotic assistance for human limb movement by detecting bioelectrical signals from extensor-flexor muscle regions $[43,44]$. Elbow flexion-extension movements that require attention to kinematic consistency were adopted as the primary task. During fNIRS recordings, healthy participants prepared for and performed the motor task in three loading conditions requiring different degrees of external attending in ascending order: non-loading condition (NON), resistive loading condition (RES), and robotic assistive loading condition (ROB). We hypothesized that by providing robotic assistance to voluntary elbow movements, the allocation of attentional resources would be biased toward external attending during motor task execution, reflecting in greater activation of rmPFC subregions during ROB compared to that during other loading conditions.

\section{Methods}

\section{Participants}

In total, 26 healthy participants ( 13 females and 13 males, age range: $22.12 \pm 1.34$ years) were enrolled in this study. Participants were right-hand dominant as assessed using the Edinburg Handedness Questionnaire (88.74 \pm 15.63$)$. None of the participants reported a medical history of neurological or psychiatric disorders, or any orthopedic injuries that impaired upper limb sensorimotor function. Participants were instructed to avoid consuming any caffeine or alcohol-containing substances for at least $12 \mathrm{~h}$ prior to experiments. Participants were informed about the study's purpose and provided written informed consent before participation. All procedures were in compliance with the Declaration of 
Helsinki and the United States Code of Federal Regulations for the protection of human participants. The present study was approved by the Human Ethics Committee of Hiroshima University (No. C-114).

\section{Apparatus}

The HAL-SJ (Cyberdyne Inc., Tsukuba, Irabaki, Japan) is a wearable assistive exoskeleton robot that can be attached to the elbow or knee to support flexion and extension joint motions. The Cybernic Voluntary Control mode in HAL-SJ provides assistive forces to facilitate joint movements based on human motion intentions for human motion detected via bioelectrical signals from muscles. In this study, the HAL-SJ was set on the lateral side of each participant's right arm with two pairs of surface electrodes attached to the muscle belly of the bicep and tricep muscles (Figure 1A). The setting parameters for HAL-SJ were standardized across participants, with an assistive gain of $45 \%$ and assistive balance between flexor and extensor motions of zero.

A self-built resistance exoskeleton device was designed to apply constant resistive force on voluntary elbow movements of the right elbow. The resistance exoskeleton device was set on the medial side of the right arm and consisted of a $1^{\circ}$-of-freedom elbow joint with two bars attached to the arm and forearm (Figure 1A). During experiments, the resistance degree imposed by the device was controlled at approximately $95 \mathrm{Nm} /$ rad for all participants.

\section{Task conditions}

Participants were seated in an upright position facing an LCD monitor (23 inches; Flexscan EV 2316V, Eizo, Japan) with a participant-monitor distance of $90 \mathrm{~cm}$. Participants placed their right elbow on an elbow-rest and aligned the right arm to the starting posture, in which the arm was in line with the horizontal plane, with a $30^{\circ}$-flexed shoulder and a $40^{\circ}$-flexed elbow (Figure 1B). A chinrest was used to limit head motion. As the present study focused on the information-processing of somatosensory feedback induced by human-robot interactions, participants were required to visually fixate on a cross displayed at the center of a monitor throughout the experiments in order to focus attention on somatosensory feedback while minimizing visual feedback during task performance. Three different loading conditions for right elbow movements were assigned to each participant in a pseudo-randomized order: non-loading condition (NON), resistive loading condition (RES), and robotic assistive loading condition (ROB). In the NON condition, elbow movements were performed without external loading. In the RES condition, a self-built resistance exoskeleton device was used to impose constant resistive force on elbow movements. In the ROB condition, elbow movements were performed using a combined configuration of HAL-SJ and the external exoskeleton device (Figure 1A), which applied assistive and resistive forces on joint motions.

\section{Task procedure}

Each session consisted of four consecutive trials in a block paradigm, as follows: Baseline period - Task period - Rest period (Figure 1C). Each task trial commenced with a baseline period, during which 
participants were required to relax their right arm for $20 \mathrm{~s}$ while baseline hemodynamic responses were recorded. We designed a 30-s task period comprising two phases: a 10-s task preparation and a 20 -s task execution. The start of the preparation phase was signaled by a verbal command " 10 seconds left" from the experimenter, during which participants were instructed to prepare for the upcoming motor task without performing any actual movements or muscle contractions. The execution phase commenced after a beep was presented. Participants were then allocated $20 \mathrm{~s}$ to perform cyclic elbow flexionextension movements with a ROM from approximately $40^{\circ}$ to $120^{\circ}$ (Figure 1B). Movement frequency was limited to 0.5 to $1 \mathrm{~Hz}$. Participants were encouraged to strive for kinematic consistency in their ROM of flexion-extension movements, referred to as motor performance. The task period ended after two beeps, which cued participants to return their arms to the starting posture and rest until the next trial.

A complete experimental session lasted approximately 4 min, with a 5-min inter-session interval for setting up the new condition. Total experimental duration was 25 min per participant. After completing experimental conditions, participants were required to complete a VAS that examined subjective measures of attentional effort (from "very low degree" to "very high degree") for each condition. Prior to participation, participants were familiarized with the task by undergoing several practice trials for each condition.

\section{Behavioral data acquisition and exclusion criteria}

To measure the absolute angle information of the forearm, a motion sensor (MPU9250; TDK InvenSense, CA, USA) was set up at the right wrist. The device featured 16-bit data outputs in the range of $\pm 2 \mathrm{G}$ for each of the three acceleration axes and $\pm 2,000 \mathrm{dps}$ (degrees per second) for each of the three gyroscopic axes, with a sampling frequency of $500 \mathrm{~Hz}$. A microcontroller (ARM mbed LPC1768; NXP Semiconductors, Eindhoven, Netherlands) input the acquired data to a Madgwick filter, calculated the attitude estimation of the absolute angle of the forearm with a sampling frequency of $20 \mathrm{~Hz}$, and sent the absolute angle information to a PC. The ROM for each cyclic elbow movement was determined by subtracting the minimal angle from the maximum angle. The SD of ROM for each trial was generated across all movements. For statistical analysis, kinematic variability in each condition was quantified by calculating the average SD values across trials.

Behavioral performance was recorded using a video recording device (iPhone; Apple Inc., CA, USA). To additionally assess the quality of interaction between voluntary movements of participants and external assistance of the HAL-SJ, the number of jerks during ROB performance was measured for each trial using visual inspection. For statistical analysis, the mean number of jerks across four trials was generated for each participant.

To verify the participant's compliance with task instructions during the experiment, their behaviors were evaluated visually and electromyographically. To monitor the muscular activity of arm muscles, bipolar surface electrodes with an inter-electrode distance of $10 \mathrm{~mm}$ were placed over the muscle belly of the right bicep and tricep muscles. A reference electrode was attached to the left wrist. EMG signals were acquired with a band-pass filter from 10 to $500 \mathrm{~Hz}$ at a sampling rate of $1 \mathrm{kHz}$ using an EMG system 
(EMG Master; Mediarea Support Business Union, Okayama, Japan). The processed EMG tracings were visually inspected to detect muscle contractions. One individual participant data with more than two trials containing incorrect behavior (i.e., marked body movements, lack of attention, noticeable muscle activity during non-execution periods) in any condition were excluded from subsequent data processing and statistical analyses. As a result, there was a total of remaining 18 participants analyzed ( 10 females and 8 males, age range: $22.11 \pm 1.49$ years).

\section{fNIRS data acquisition}

A multi-channel fNIRS system (FOIRE-3000; Shimadzu, Kyoto, Japan) was employed to measure cortical hemodynamic activity during experiments with a sampling rate of $7.69 \mathrm{~Hz}$. Specifically, the measured changes in light absorption recorded at three wavelengths $(780,805$, and $830 \mathrm{~nm})$ via semiconductor laser diodes were transformed into corresponding concentration changes in oxy- $\mathrm{Hb}$, deoxy- $\mathrm{Hb}$, and total hemoglobin (total-Hb) using the modified Lambert-Beer law [61]. These values were measured using the unit of molar concentration multiplied by length $(\mathrm{mM} \times \mathrm{mm})$. Given that changes in oxy- $\mathrm{Hb}$ signal are the most sensitive indicator of changes in regional cortical blood flow and have the highest signal-to-noise ratio $[45,46]$, the analysis and discussion in this study focused primarily on changes in oxy- $\mathrm{Hb}$ concentration.

Optodes were fixed to each participant's scalp using a customized head cap with a 30-probe layout (14 sources and 16 detectors) (Figure 3A). Optical probes comprised 42 long-separation channels (with a 3$\mathrm{cm}$ source-detector distance), covering cerebral cortical regions including the rmPFC, dIPFC, PM, M1, and S1 (Figure 3B). As the fNIRS signal is derived from both regional cortical blood flow and scalp blood flow [62], oxy- $\mathrm{Hb}$ data from four short-separation channels (with a 1.5-cm source-detector distance) were included in the analysis in order to separate cortex-derived hemodynamic responses from global physiological changes in the scalp. To remove the effects of scalp blood flow on signals, each long channel was regressed with the closest short channel. Probes were placed according to the international 10-20 electroencephalogram electrode system, with the midline central point of the scalp (Cz) positioned beneath the $12^{\text {th }}$ channel. To obtain channel-related anatomical information, a 3D digitizer (FASTRAK, Polhemus, Colchester, Vermont, USA) was used to record the 3-dimensional position of each optical probe and four reference landmarks including nasion, $\mathrm{Cz}$, left auricular, and right auricular points. Channel locations were estimated from coordinates of optodes and reference points using the Montreal Neurological Institute standard space coordinates. To anatomically label fNIRS channels, probabilistic mapping between each fNIRS channel and its corresponding BA was performed using the open-source software package NIRS-SPM (BISP Lab, Daejeon, Korea) implemented in MATLAB (MathWorks, Natick, Massachusetts, USA). The channel sets for ROls were selected based on BAs and anatomical locations of cortical areas for each participant (Table 1). To investigate neural signals in subregions of the rmPFC, channels within BA 10 were classified into two subdivisions along the ventral-dorsal axis: the ventral rmPFC, located in the ventral-middle part of BA 10 adjacent to BA 11 (orbital prefrontal cortex); and the dorsal rmPFC, positioned in the dorsal-middle part of BA 10 adjacent to BA 9 (dorsolateral prefrontal cortex). 
fNIRS signals were first processed based on moving standard deviation and spline interpolation methods to detect and reduce motion artifacts. A band-pass filter with a $0.01-0.1 \mathrm{~Hz}$ cutoff frequency range was then applied to remove concomitant systemic responses from the signal. The oxy-Hb time-series in each channel were corrected to baseline values, determined as the mean over $2 \mathrm{~s}$ prior to the onset of the task period. Subsequently, the oxy- $\mathrm{Hb}$ time-series were averaged across trials and ROI-wise channels to generate ROI time-series for each condition. Based on the ROI time-series, mean oxy-Hb changes were used as an index of cortical activation and were calculated separately for each task phase, namely the preparation phase ( 0 to $10 \mathrm{~s}$ ) and execution phase ( 15 to $35 \mathrm{~s}$ ), with the task onset set at $0 \mathrm{~s}$. The time window for the execution phase was defined based on the temporal characteristics of blood oxygenation hemodynamic responses.

Based on a report by Schroeter et al. [47], we employed effect size as an index of cortical activation due to its robustness to differential path-length factors. For each channel, the effect size in each execution period was calculated as the difference between the mean oxy-Hb changes in the execution window (15 to $35 \mathrm{~s}$ ) and baseline window (-5 to $5 \mathrm{~s}$ ), divided by the SDs of the baseline window. For statistical analysis, the effect sizes were averaged across trials and ROI-wise channels to generate the average effect sizes for each condition.

fNIRS data were analyzed using NIRS-SPM [48]. Wavelet-minimum description length (Wavelet-MDL) detrending algorithm was applied to the data to exclude trends related to breathing, cardiovascular responses, and other experimental noise [63]. The mass univariate method based on the general linear model in NIRS-SPM was used to create a model of theoretical responses according to task design. Three comparisons were investigated: ROB versus RES, ROB versus NON, and RES versus NON. The general linear model method compared the theoretical model with actual hemodynamic responses to calculate tstatistics for each channel. Group-level t-statistic maps were generated to determine execution-related activation for each comparison at a p-value less than $0.0167(0.05 / 3)$ by Bonferroni correction. Alignment of analysis results based on hemodynamic responses, effect sizes, and t-statistic maps would provide evidence of cortical activity.

\section{Statistical analysis}

One-way repeated-measures ANOVA tests were used to examine the effect of condition (ROB, RES, and NON) on indices of cortical activation (mean oxy-Hb changes and average effect size) for each ROI. Bonferroni post-hoc tests were applied for multiple comparisons. To examine the association between cortical activity and task performance, Pearson's correlation analysis was performed for execution-related oxy-Hb responses in rmPFC subregions and kinematic variability. SPSS statistical package version 19.0 (IBM, Co. Ltd, New York, USA) was used for statistical analysis. P-values less than or equal to 0.05 were considered statistically significant.

\section{Results}


To address our hypotheses, we conducted a human fNIRS experiment where participants were required to execute the motor task in which right elbow movements were made both with and without robotic assistance. Total 26 right-handed healthy participants engaged in the present study.

Participants were seated facing an LCD monitor, with their right arm resting upon a table (Figure 1B). Three different loading conditions for right elbow movements were assigned to each participant in a pseudo-randomized order: non-loading condition (NON), resistive loading condition (RES), and robotic assistive loading condition (ROB). In the NON condition, elbow movements were performed without external loading, demanding minimal operation of external attending. In the RES condition, a self-built resistance exoskeleton device was set on the right arm to impose constant resistive force on elbow movements. The involvement of the external attending in RES was greater than that in NON. In the ROB condition, participants carried out elbow movements in a combined configuration of the HAL-SJ and the external exoskeleton device (Figure 1A). The HAL-SJ was set to the Cybernic Voluntary Control mode which allows it to provide real-time external assistance to elbow movements by detecting human motion intention via bioelectrical signals from muscles. The heavy reliance on somatosensory feedback for human-robot coordination during ROB warranted the highest degree of external attending among the three conditions, therefore requiring greater rmPFC activation to generate attentional bias toward external information-processing.

For each condition, participants performed 4 consecutive trials in a block paradigm, as follows: Baseline period - Task period - Rest period (Figure 1C). Each trial began with a 20-s period requiring participants to relax their right arm while baseline hemodynamic responses were recorded. We designed a 30-s task period comprising two phases: a 10-s task preparation and a 20 -s task execution, using separate starting auditory signals. During task preparation, participants were instructed to prepare for the upcoming motor task without performing any actual movements or muscle contractions. During task execution, participants had to perform cyclic elbow flexion-extension movements. Participants were asked to strive for kinematic consistency in range-of-motion (ROM) smoothly and reduce the number of jerks, referred to as motor performance. Subjective measures of attentional effort were reported on a visual analog scale (VAS), immediately after the completion of each condition. One individual participant data with more than two trials containing incorrect behavior (see the methods) in any condition were excluded from further analysis, resulting in a total of 18 participants analyzed.

\section{Behavioral results}

We analyzed participants' performance in terms of the kinematic variability in ROM for elbow movements using a motion sensor that measures the absolute angle information of the forearm. The standard deviation (SD) of ROM for each trial was analyzed across all movements, then averaged across trials to obtain the index of kinematic variability for each trial.

One-way repeated measures ANOVA revealed significant differences in kinematic variability among conditions $\left(F_{2,34}=3.364, p=0.046\right.$, Figure 2). Post-hoc testing using Bonferroni correction revealed that 
kinematic variability was significantly higher during ROB than during RES $(p=0.033)$.

\section{fNIRS results}

To assess task-related cortical activity, we used a multi-channel fNIRS system (FOIRE-3000) to monitor concentration changes in oxy- $\mathrm{Hb}$ and deoxy-Hb for each participant. Only oxy-Hb concentration $(\mathrm{mM} \times \mathrm{mm})$ was adopted for analyses. A customized head cap with a 30-fNIRS-probe layout was fixed to each participant's scalp (Figure 3A), and each long channel was regressed with the closest short channel. The resulting 42 fNIRS channels approximately evenly covered the ventral and dorsal part of rmPFC, the bilateral dorsolateral prefrontal cortex (dIPFC), premotor area (PM), primary motor cortex (M1), and primary somatosensory cortex (S1) (Figure 3B). These cortical regions were determined as regions of interest (ROI).

For each condition, fNIRS time-series data corrected for the baseline (the 2-s interval prior to the task onset) were averaged across trials and ROI-wise channels to generate ROI time-series data. Accordingly, preparation-related, and execution-related $\mathrm{oxy}-\mathrm{Hb}$ changes were calculated using separate analyzing time windows (task preparation: 0 to $10 \mathrm{~s}$; task execution: 15 to $35 \mathrm{~s}$ ), with the task onset set at $0 \mathrm{~s}$. For illustration, grand-average ROI time-series of oxy- $\mathrm{Hb}$ responses in the ventral rmPFC during ROB, RES, and NON are presented in Figure 3C.

With regards to preparation-related oxy-Hb changes, one-way repeated measures ANOVAs revealed significant differences in oxy-Hb responses among conditions in the left dIPFC $\left(F_{2,34}=4.28, p=0.022\right)$ (Figure 4A). Bonferroni post-hoc testing revealed that oxy-Hb responses in the left dIPFC were significantly higher during ROB and RES than during NON ( $p=0.039$ and $p=0.012$, respectively).

With regards to execution-related oxy-Hb changes, one-way repeated measures ANOVA revealed significant differences in oxy-Hb responses among conditions in the three ROIs (Figure 4B): ventral $\operatorname{rmPFC}\left(F_{2,34}=22.10, p<0.0001\right)$, dorsal $\operatorname{rmPFC}\left(F_{2,34}=11.26, p<0.0001\right)$, and right $P M\left(F_{2,34}=5.80, p=\right.$ 0.007). $0 x y-H b$ responses in the ventral rmPFC region were stronger during ROB than during RES $(p=$ $0.002)$ and NON $(p<0.0001)$. Cortical activation in the ventral rmPFC was significantly greater during RES than during NON $(p=0.020)$, whereas activity in the dorsal rmPFC was significantly greater during ROB than during NON $(p=0.001)$. Oxy-Hb responses in the right PM were significantly higher during RES than during NON $(p=0.005)$.

Furthermore, we analyzed execution-related cortical activity based on effect sizes, which are robust to different path-length factors across participants and fNIRS channels [47]. The effect sizes were averaged across trials and ROI-wise channels to generate the average effect sizes for each condition. One-way repeated measures ANOVA revealed a significant main effect of loading condition on the effect size of hemodynamic changes in the ventral rmPFC (ROB: $4.07 \pm 1.11$, RES: $0.28 \pm 1.02$, NON: $-2.26 \pm 0.81 ; F_{2,34}$ $=17.872, p<0.0001$ ), dorsal rmPFC (ROB: $2.76 \pm 0.68$, RES: $-1.18 \pm 1.08$, NON: $-2.08 \pm 0.87 ; F_{2,34}=10.74$, $p<0.0001$ ), and right PM (ROB: $0.83 \pm 0.64$, RES: $1.97 \pm 0.43$, NON: $-0.46 \pm 0.54 ; F_{2,34}=6.942, p=0.003$ ). 
In ventral and dorsal rmPFC regions, significantly greater effect sizes were observed in ROB compared to those in RES (ventral rmPFC: $p=0.019$, dorsal rmPFC: $p=0.011$ ) and NON (ventral rmPFC: $p<0.0001$, dorsal rmPFC: $p<0.0001)$. In the ventral rmPFC, a significantly greater effect size was observed in RES compared to that in NON $(p=0.040)$. In the right $P M$, the effect size was significantly greater during RES than during NON $(p=0.001)$.

The fNIRS data were also analyzed using General Linear Model-based approaches in Statistical Parametric Mapping for NIRS (NIRS-SPM) [48], in which actual hemodynamic responses were compared to models of theoretical responses to calculate t-statistics for each channel. Three comparisons were investigated: ROB versus RES, ROB versus NON, and RES versus NON. Group-level t-statistic maps were generated to determine execution-related activation for each comparison at a p-value less than 0.0167 $(0.05 / 3)$ by Bonferroni correction. Group analysis of the comparison between ROB and NON revealed significant activity in both the ventral and dorsal parts of the rmPFC (Figure $5 \mathrm{~A}$ ). For the comparison between ROB and RES, increased cortical activation was specifically identified in the ventral rmPFC (Figure 5B).

\section{Correlation results}

Correlation analyses of the relationship between fNIRS data of the ventral and dorsal rmPFC and motor performance revealed that execution-related oxy-Hb responses in the dorsal rmPFC during ROB were positively correlated with kinematic variability $(r=0.523, p=0.026)$ (Figure 6). Additionally, no significant association was observed between cortical activity and behavioral measures including VAS scores and the number of jerks in ROB.

\section{Discussion}

The present study aimed to investigate the effects of robot-assisted execution on cortical hemodynamic responses in the rmPFC using fNIRS in healthy participants. We hypothesized that human-robot interactions in ROB would activate rmPFC subregions to bias attentional resource allocation toward external information-processing. Statistical analyses of hemodynamic changes and effect sizes revealed greater activation in both the ventral and dorsal rmPFC during ROB compared to that during NON. Further, activity in the ventral rmPFC was greater during ROB than during RES. These findings aligned with the results of group-level NIRS-SPM t-statistic maps. Notably, hemodynamic responses in the dorsal rmPFC during ROB execution were positively correlated with kinematic variability.

In the present study, both ventral and dorsal parts of the rmPFC exhibited stronger activation during ROB compared to that during NON. Based on the gating hypothesis proposed by Burgess and colleagues $[15,49,50]$, the SAG system acts as a gateway between internal and external attending. This system monitors and generates attentional bias toward the relevant attending mode for optimizing behavioral responses. Studies examining this hypothesis have reported that subregions of the rostral PFC contribute substantially to processing in the SAG system $[15,31,36,50]$. Specifically, the rIPFC predominantly biases internal attending, whereas the rmPFC mainly prioritizes external attending. Further, the rmPFC exhibits 
functional coordination with the striatum and thalamus when an individual engages in external attending [31]. These subcortical regions are known to modulate sensory information-processing to the PFC $[32,33]$. Therefore, our findings provide support for the gating hypothesis and concur with previous evidence, suggesting that greater hemodynamic responses in the rmPFC during ROB reflect high-level attentional control that biases the allocation of attentional resources toward external information-processing.

We observed that cortical activity in the ventral rmPFC was significantly greater during ROB relative to that during RES. Analysis of the behavioral data revealed higher kinematic variability during ROB compared to that during RES, indicating considerable demands for attentional control for human-robot interactions. These findings are consistent with previous reports of increased activation in the ventral rmPFC during conditions requiring a high degree of external attending [31,36,37]. In a neuroimaging study by Henseler et al. [31], participants were scanned using functional magnetic resonance imaging (fMRI) while performing finger tapping tasks in three conditions, which required direction of attention toward an external cue, internal cue, or both. The authors reported that the most anterior parts of bilateral rmPFC regions, corresponding to the ventral rmPFC in our study, were specifically activated during the condition that highly involved external attending. Henseler et al. [31] conjectured that the ventral rmPFC plays a key role in cognitive processes that generate attentional bias toward external attending when task performance is strongly influenced by sensory feedback. Furthermore, a posterior-to-anterior organization in hierarchical control of cognition by the PFC has been proposed [51,52]. Under this framework, progressively anterior subdivisions of the PFC operate as higher-order structures in the hierarchy which represent progressively information-processing aspects of cognition and deploy top-down control over more posteriorly located regions. In this regard, our results suggest that the ventral rmPFC acts as a higher-level node in cognitive processes which exerts top-down attentional control over more dorsally located regions to bias the allocation of attentional resources toward external information-processing during human-robot interactions.

Our correlation analysis revealed that hemodynamic changes in the dorsal rmPFC during ROB execution were positively correlated with kinematic variability. This finding is consistent with previously reported evidence of significant associations between cortical activity in the rmPFC and task performance [31,5355]. Supervisory attentional processes of the rmPFC are thought to operate based on the determination of the relative relationship between the current allocation of attentional resources and the attentional demand to produce optimal behavior [15]. As such, these processes may be associated with ongoing motor behavior, given that less optimal performance may induce greater top-down attentional bias toward the attending mode relevant to the current motor goal. Moreover, recent fNIRS evidence has indicated that rmPFC-related cognitive processes modulate neural plasticity in sensorimotor cortical regions during motor learning in physical [39] or neurofeedback configurations [56,57]. Therefore, our findings complement the evidence for an interaction between ongoing motor behavior and corresponding top-down rmPFC activity that governs the allocation of attentional resources toward external informationprocessing. 
We did not observe any significant differences in preparatory activity in the rmPFC among conditions. Preparing for impending effortful tasks engages internal or stimulus-independent cognition whereby the brain exerts top-down control to proactively mobilize attentional resources in order to ensure successful performance in the absence of external stimuli. In contrast, supervisory attentional processes of the rmPFC operate based on an external or stimulus-oriented cognition [15], in which stimulation from distal sensory modalities is required [14]. Thus, our findings further support the hypothesis that executionrelated activity in the rmPFC reflects executive attentional control that involves the processing of external information.

These study results provide potential practical implications for the improvement of robot-assisted rehabilitation. Our findings showed that kinematic engagement in a robot-assisted task involved greater rmPFC activation relevant to executive attentional control than those in other manual loading tasks. It has been proved that increased attentional control may correlate with the enhancement of active engagement in task performance [58]. Here, the active assistive exoskeleton robot provided external assistance to arm movements by detecting and responding to human motion intention. The application of intention-based assistance has been suggested to reduce slacking and engage subjects more actively in therapeutic training $[59,60]$. In the present study, participants were required to strive for kinematic consistency in their movements during human-robot interactions, instead of simply executing with robotic assistance. Combining robot-assisted therapies with a task-oriented approach may encourage subjects to make further efforts to efficiently interact with robotic assistance, involving both motor learning and active engagement. We suggest that this type of robot-assisted task-oriented therapy may be effective in promoting specific processes of top-down attentional control during therapeutic training, potentially facilitating neurological functional recovery.

The present study has several limitations. First, the setting parameters for HAL-SJ were standardized across all participants without consideration of individual kinematic characteristics. Future studies should consider personalized adjustment of robotic assistance. Second, fNIRS technology has a low spatial resolution of $3 \mathrm{~cm}^{\prime} 3 \mathrm{~cm}$. In the present study, we focused on cortical oxy-Hb responses in largescale cortical regions. It would be desirable for future studies to employ a highly dense fNIRS probe set in order to obtain more precise information about task-related neural signals. The third technical limitation of fNIRS is that it is only capable of measuring cartical signals from the cortical layer of prefrontal regions. As such, hemodynamic signals in subdivisions lying deep under the cerebral surface are undetectable by fNIRS. Future studies should also consider harnessing multi-modal neuroimaging measurements such as fNIRS-fMRI recordings.

In conclusion, the current study revealed the profile of cortical hemodynamic activity in the rmPFC during motor execution with an assistive exoskeleton robot. Increased hemodynamic responses in the rmPFC, especially its ventral part, were observed when participants were engaged in kinematic human-robot interactions. rmPFC cortical activation may reflect top-down attentional control to bias the allocation of attentional resources toward external information-processing which is necessary for task performance 
with robotic assistance. Our study provides crucial insight into the neural mechanisms underlying robotassisted execution and rehabilitative interventions.

\section{Declarations}

\section{Data availability}

The datasets analyzed during the current study are available from the corresponding author on a reasonable request.

\section{Acknowledgements}

This work is supported by Hiroshima ROBOCARE CENTER, and I would like to thank Akiko Araki, a director of the center.

\section{Author contributions}

D TL, KW, HO, NF and SU performed experiments; KM, NI, ST, YI and TI supported technically; TI, TO, HN, NF and SU interpreted results of experiments; D TL and SU prepared figures and table; D TL, NF and SU drafted manuscript; D TL, KM, TI, HA, OA, TO, HN, NF and SU approved final version of manuscript; D TL, $\mathrm{HA}, \mathrm{OA}, \mathrm{TO}, \mathrm{HN}, \mathrm{NF}$ and SU edited and revised manuscript.

\section{Additional information}

Competing interests: The authors declare no competing interests.

\section{References}

1. Lozano, R. et al. Global and regional mortality from 235 causes of death for 20 age groups in 1990 and 2010: a systematic analysis for the Global Burden of Disease Study 2010.2095-2128(2010).

2. Sivan, M., O'Connor, R. J., Makower, S., Levesley, M. \& Bhakta, B. Systematic review of outcome measures used in the evaluation of robot-assisted upper limb exercise in stroke. J. Rehabil. Med. 43, 181-189 (2011).

3. Turner, D. L., Ramos-Murguialday, A., Birbaumer, N., Hoffmann, U. \& Luft, A. Neurophysiology of robotmediated training and therapy: A perspective for future use in clinical populations. Front. Neurol. 4 NOV, 1-12 (2013).

4. Calabrò, R. S. et al. Robotic gait rehabilitation and substitution devices in neurological disorders: where are we now? Neurol. Sci. 37, 503-514 (2016).

5. Godfrey, S. B., Holley, R. J. \& Lum, P. S. Clinical effects of using HEXORR (Hand Exoskeleton Rehabilitation Robot) for movement therapy in stroke rehabilitation. Am. J. Phys. Med. Rehabil. 92, 947-958 (2013). 
6. Klamroth-Marganska, V. et al. Three - dimensional, task - specific robot therapy of the arm : a multicenter randomized clinical trial in stroke patients. Zurich Open Repos. Arch. 13, 159-166 (2014).

7. Sale, P. et al. Recovery of hand function with robot-assisted therapy in acute stroke patients: A randomized-controlled trial. Int. J. Rehabil. Res. 37, 236-242 (2014).

8. Takahashi, K. et al. Efficacy of Upper Extremity Robotic Therapy in Subacute Poststroke Hemiplegia: An Exploratory Randomized Trial. Stroke. 47, 1385-1388 (2016).

9. Chun, M. M. \& Golomb, J. D. \& Turk-Browne, N. B. A Taxonomy of external and internal attention. Annu. Rev. Psychol. 62, 73-101 (2011).

10. Lieberman, M. D. Social cognitive neuroscience: A review of core processes. Annu. Rev. Psychol. 58, 259-289 (2007).

11. Andrews-Hanna, J. R. The brain's default network and its adaptive role in internal mentation. Neuroscientist. 18, 251-270 (2012).

12. Buckner, R. L., Andrews-Hanna, J. R. \& Schacter, D. L. The brain's default network: Anatomy, function, and relevance to disease. Ann. N. Y. Acad. Sci. 1124, 1-38 (2008).

13. Miller, E. K. \& Cohen, J. D. An integrate theory of PFC function. Annu. Rev. Neurosci. 24, 167-202 (2001).

14. Dixon, M. L., Fox, K. C. R. \& Christoff, K. A framework for understanding the relationship between externally and internally directed cognition. Neuropsychologia. 62, 321-330 (2014).

15. Burgess, P. W., Dumontheil, I. \& Gilbert, S. J. The gateway hypothesis of rostral prefrontal cortex (area 10) function. Trends Cogn. Sci. 11, 290-298 (2007).

16. Sigrist, R., Rauter, G., Riener, R. \& Wolf, P. Augmented visual, auditory, haptic, and multimodal feedback in motor learning: A review. Psychon. Bull. Rev. 20, 21-53 (2013).

17. Simonetti, D., Zollo, L., Papaleo, E., Carpino, G. \& Guglielmelli, E. Multimodal adaptive interfaces for 3D robot-mediated upper limb neuro-rehabilitation: An overview of bio-cooperative systems. Rob. Auton. Syst. 85, 62-72 (2016).

18. Beckerle, P. et al. A human-robot interaction perspective on assistive and rehabilitation robotics. Front. Neurorobot. 11, 1-6 (2017).

19. Maeda, R. S., Cluff, T., Gribble, P. L. \& Pruszynski, J. A. Feedforward and feedback control share an internal model of the arm's dynamics. J. Neurosci. 38, 10505-10514 (2018).

20. Saita, K. et al. Combined therapy using botulinum toxin $A$ and single-joint hybrid assistive limb for upper-limb disability due to spastic hemiplegia. J. Neurol. Sci. 373, 182-187 (2017).

21. Saita, K. et al. Biofeedback effect of hybrid assistive limb in stroke rehabilitation: A proof of concept study using functional near infrared spectroscopy. PLoS One. 13, 1-12 (2018).

22. Berger, A., Horst, F., Müller, S., Steinberg, F. \& Doppelmayr, M. Current state and future prospects of EEG and fNIRS in robot-assisted gait rehabilitation: A brief review. Front. Hum. Neurosci.13, (2019). 
23. Kim, H. Y., Yang, S. P., Park, G. L., Kim, E. J. \& You, J. H. Best facilitated cortical activation during different stepping, treadmill, and robot-assisted walking training paradigms and speeds: A functional near-infrared spectroscopy neuroimaging study. NeuroRehabilitation. 38, 171-178 (2016).

24. Simis, M., Santos, K., Sato, J., Fregni, F. \& Battistella, L. T107. Using Functional near Infrared Spectroscopy (fNIRS) to assess brain activity of spinal cord injury patient, during robot-assisted gait. Clin. Neurophysiol. 129, e43-e44 (2018).

25. Koechlin, E., Basso, G., Pietrini, P., Panzer, S. \& Grafman, J. The role of the anterior prefrontal cortex in human cognition. Nature. 399, 148-151 (1999).

26. Ramnani, N. \& Owen, A. M. Anterior prefrontal cortex: Insights into function from anatomy and neuroimaging. Nat. Rev. Neurosci. 5, 184-194 (2004).

27. Mansouri, F. A., Koechlin, E., Rosa, M. G. P. \& Buckley, M. J. Managing competing goals - a key role for the frontopolar cortex. Nat. Publ. Gr. 18, 645-657 (2017).

28. Petrides, M. \& Pandya, D. N. Petrides \& Pandya PFC. Eur J Neurosci. 11, 1-26 (1999).

29. Morán, M. A., Mufson, E. J. \& Mesulam, M. M. Neural inputs into the temporopolar cortex of the rhesus monkey. J. Comp. Neurol. 256, 88-103 (1987).

30. Arikuni, T., Sako, H. \& Murata, A. Ipsilateral connections of the anterior cingulate cortex with the frontal and medial temporal cortices in the macaque monkey. Neurosci. Res. 21, 19-39 (1994).

31. Henseler, I., Krüger, S., Dechent, P. \& Gruber, O. A gateway system in rostral PFC? Evidence from biasing attention to perceptual information and internal representations. Neuroimage. 56, 16661676 (2011).

32. Frank, M. J., Loughry, B. \& Reilly, R. C. O. Frank_2001_Interactions. 1, 137-160 (2001).

33. O’Reilly, R. C. \& Frank, M. J. Making working memory work: A computational model of learning in the prefrontal cortex and basal ganglia. Neural Comput. 18, 283-328 (2006).

34. Christoff, K. \& Gabrieli, J. D. E. The frontopolar cortex and human cognition: Evidence for a rostrocaudal hierarchical organization within the human prefrontal cortex. Psychobiology. 28, 168186 (2000).

35. Badre, D. \& Nee, D. E. Frontal Cortex and the Hierarchical Control of Behavior. Trends Cogn. Sci. 22, 170-188 (2018).

36. Gilbert, S. J. et al. Distinct regions of medial rostral prefrontal cortex supporting social and nonsocial functions. Soc. Cogn. Affect. Neurosci. 2, 217-226 (2007).

37. Simons, J. S., Henson, R. N. A., Gilbert, S. J. \& Fletcher, P. C. Separable forms of reality monitoring supported by anterior prefrontal cortex. J. Cogn. Neurosci. 20, 447-457 (2008).

38. Schytz, H. W. et al. A new technology for detecting cerebral blood flow: A comparative study of ultrasound tagged NIRS and 133Xe-SPECT. Neurocrit. Care. 17, 139-145 (2012).

39. Ishikuro, K., Urakawa, S., Takamoto, K., Ishikawa, A. \& Ono, T. Cerebral functional imaging using nearinfrared spectroscopy during repeated performances of motor rehabilitation tasks tested on healthy subjects. 8,1-13(2014). 
40. Mihara, M., Miyai, I., Hatakenaka, M., Kubota, K. \& Sakoda, S. Sustained prefrontal activation during ataxic gait: A compensatory mechanism for ataxic stroke ? 37,1338-1345(2007).

41. Urakawa, S., Takamoto, K., Ishikawa, A., Ono, T. \& Nishijo, H. Selective Medial Prefrontal Cortex Responses During Live Mutual Gaze Interactions in Human Infants: An fNIRS Study. Brain Topogr. 28, 691-701 (2015).

42. Sankai, Y. \& Sakurai, T. Exoskeletal cyborg-type robot. 3912, 2-4 (2018).

43. Morishita, T. \& Inoue, T. Interactive bio-feedback therapy using hybrid assistive limbs for motor recovery after stroke: Current practice and future perspectives. Neurol. Med. Chir. (Tokyo). 56, 605612 (2016).

44. Suzuki, K., Mito, G., Kawamoto, H., Hasegawa, Y. \& Sankai, Y. Intention-Based Walking Support for Paraplegia Patients with Robot Suit HAL. Intech. 13, https://doi.org/10.5772/57353 (2016).

45. Hoge, R. D. et al. Simultaneous recording of task-induced changes in blood oxygenation, volume, and flow using diffuse optical imaging and arterial spin-labeling MRI. Neuroimage. 25, 701-707 (2005).

46. Okamoto, M. et al. Multimodal assessment of cortical activation during apple peeling by NIRS and fMRI. Neuroimage. 21, 1275-1288 (2004).

47. Schroeter, M. L., Zysset, S. \& Kruggel, F. \& Von Cramon, D. Y. Age dependency of the hemodynamic response as measured by functional near-infrared spectroscopy. Neuroimage. 19, 555-564 (2003).

48. Ye, J. C., Tak, S., Jang, K. E., Jung, J. \& Jang, J. NIRS-SPM: Statistical parametric mapping for nearinfrared spectroscopy. Neuroimage. 44, 428-447 (2009).

49. Burgess, P. W., Gilbert, S. J. \& Dumontheil, I. Function and localization within rostral prefrontal cortex (area 10). Philos. Trans. R. Soc. B Biol. Sci. 362, 887-899 (2007).

50. Burgess, P. W. \& Wu, H. C. Rostral prefrontal cortex (Brodmann area 10): metacognition in the brain.Princ. Front. Lobe Funct.524-534(2013).

51. Rahnev, D. Top-Down Control of Perceptual Decision Making by the Prefrontal Cortex. Curr. Dir. Psychol. Sci. 26, 464-469 (2017).

52. Koechlin, E. \& Summerfield, C. An information theoretical approach to prefrontal executive function. Trends Cogn. Sci. 11, 229-235 (2007).

53. Gilbert, S. J., Simons, J. S., Frith, C. D. \& Burgess, P. W. Performance-related activity in medial rostral prefrontal cortex (area 10) during low-demand tasks. J. Exp. Psychol. Hum. Percept. Perform. 32, 45-58 (2006).

54. Gilbert, S. J. et al. Functional specialization within rostral prefrontal cortex (area 10): A metaanalysis. J. Cogn. Neurosci. 18, 932-948 (2006).

55. Small, D. M. et al. The posterior cingulate and medial prefrontal cortex mediate the anticipatory allocation of spatial attention. Neuroimage. 18,633-641 (2003).

56. Ota, Y. et al. Motor Imagery Training With Neurofeedback From the Frontal Pole Facilitated Sensorimotor Cortical Activity and Improved Hand Dexterity. Front. Neurosci. 14, 1-16 (2020). 
57. Kinoshita, A. et al. Development of a neurofeedback protocol targeting the frontal pole using nearinfrared spectroscopy. Psychiatry Clin. Neurosci. 70, 507-516 (2016).

58. Danzl, M. M., Etter, N. M., Andreatta, R. O. \& Kitzman, P. H. Facilitating neurorehabilitation through principles of engagement. J. Allied Health. 41, 35-41 (2012).

59. Washabaugh, E. P., Treadway, E., Gillespiec, B., Remy, R. D., Krishnan, C. \& C. \& Self-powered robots to reduce motor slacking during upper-extremity rehabilitation: A proof of concept study. Restor. Neurol. Neurosci. 36, 693-708 (2018).

60. Blank, A. A., French, J. A., Pehlivan, A. U. \& O’Malley, M. K. Current Trends in Robot-Assisted UpperLimb Stroke Rehabilitation: Promoting Patient Engagement in Therapy. Curr. Phys. Med. Rehabil. Reports. 2, 184-195 (2014).

61. Delpy, D. T. et al. Estimation of optical pathlength through tissue from direct time of flight measurement. Phys. Med. Biol. 33, 1433-1442 (1988).

62. Takahashi, T. et al. Influence of skin blood flow on near-infrared spectroscopy signals measured on the forehead during a verbal fluency task. Neuroimage. 57, 991-1002 (2011).

63. Jang, K. E. et al. Wavelet minimum description length detrending for near-infrared spectroscopy. J. Biomed. Opt. 14, 034004 (2009).

\section{Tables}

Table 1. Anatomically labeled fNIRS channel locations using Brodmann areas.

The channel sets for ROls were individually adjusted for each participant based on NIRS-SPM probabilistic mapping. 


\begin{tabular}{|llll|}
\hline Channels & $\begin{array}{l}\text { Brodmann areas } \\
\text { (BA) }\end{array}$ & Regions of interest (ROI) & Abbreviation \\
\hline 1,3 & BA 1, 2, 3 & Right primary somatosensory cortex & Right S1 \\
\hline 2,5 & BA 1, 2, 3 & Left primary somatosensory cortex & Left S1 \\
\hline $6,7,10$ & BA 4 & Right primary motor cortex & Right M1 \\
\hline $8,9,14$ & BA 4 & Left primary motor cortex & Left M1 \\
\hline $11,15,16$, & BA 6 & $\begin{array}{l}\text { Right premotor and supplementary motor } \\
\text { cortex }\end{array}$ & Right PM \\
\hline $19,17,18$, & BA 6 & $\begin{array}{l}\text { Left premotor and supplementary motor } \\
\text { cortex }\end{array}$ & Left PM \\
\hline 23 & Right dorsolateral prefrontal cortex & Right dIPFC \\
\hline $28,31,33$ & BA 9,46 & Left dorsolateral prefrontal cortex & Left dIPFC \\
\hline $30,32,35$ & BA 9,46 & Dorsal rostromedial prefrontal cortex & $\begin{array}{l}\text { Dorsal } \\
\text { rmPFC }\end{array}$ \\
\hline $34,36,37$ & BA 10 & Ventral rostromedial prefrontal cortex & Ventral \\
\hline $39,31,32$ & BA 10 & & rmPFC \\
\hline
\end{tabular}

\section{Figures}




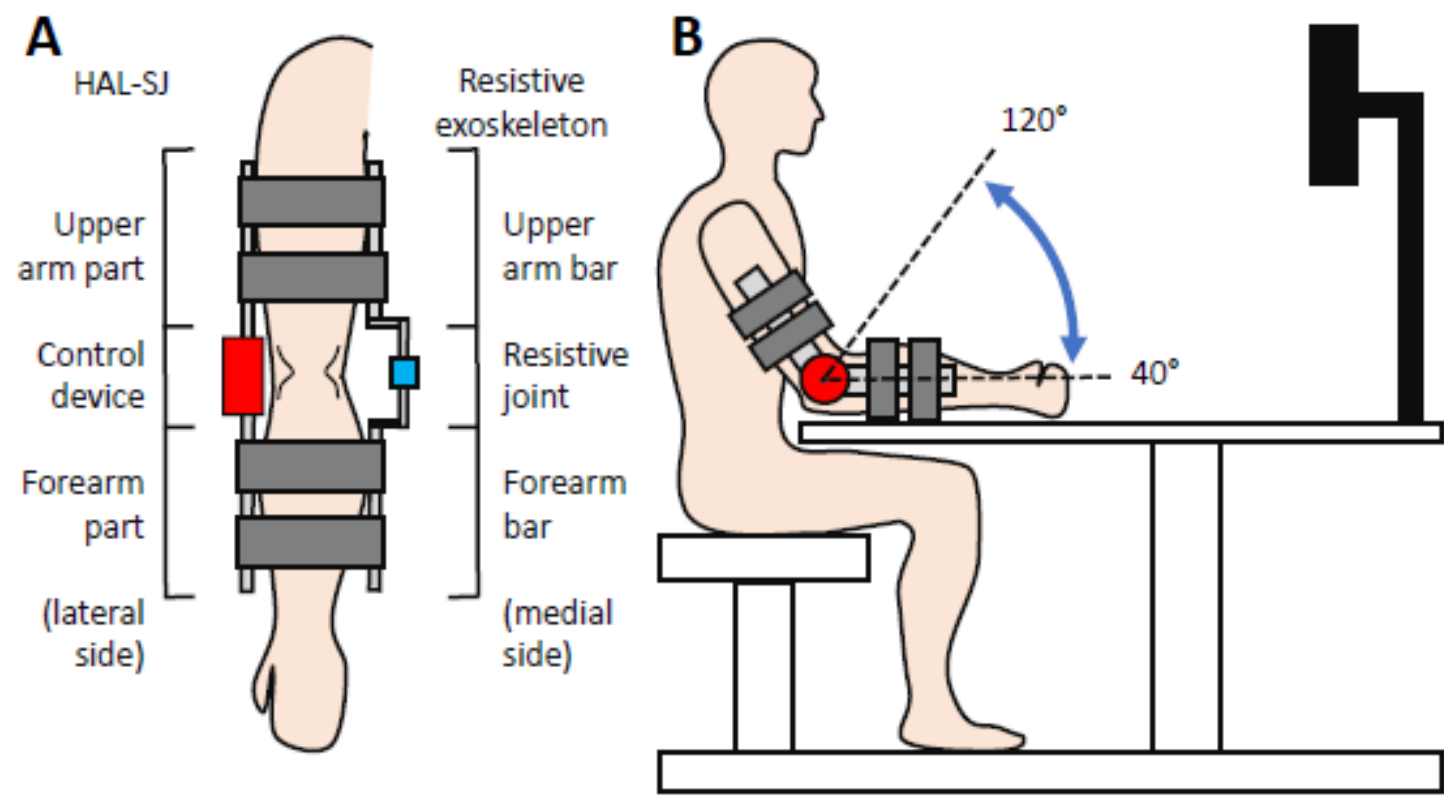

C

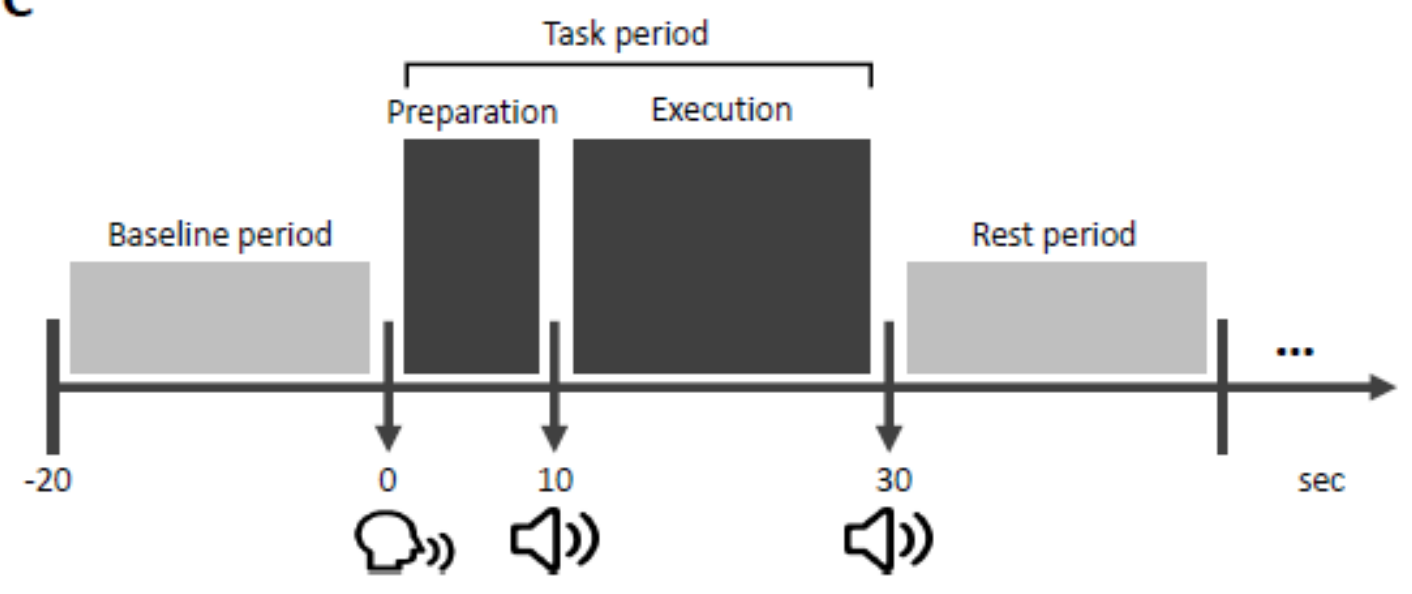

\section{Figure 1}

Experimental setup. (A) The HAL-SJ and the resistive exoskeleton device were mounted on the lateral and medial sides of the right arm, respectively. (B) Experimental setup from a sagittal view. During the execution phase of the task period, participants were required to perform cyclic elbow flexion-extension movements with the ROM from 40 to 120 degrees (dash line). (C) Experimental trial protocol. The simplified scheme of the experimental trial protocol. Each task period was $30 \mathrm{~s}$, including a 10-s preparation phase and a 20-s execution phase. The post-task period was pseudorandomized between 20 $\mathrm{s}, 30 \mathrm{~s}$, and $40 \mathrm{~s}$. ROM, range-of-motion. 


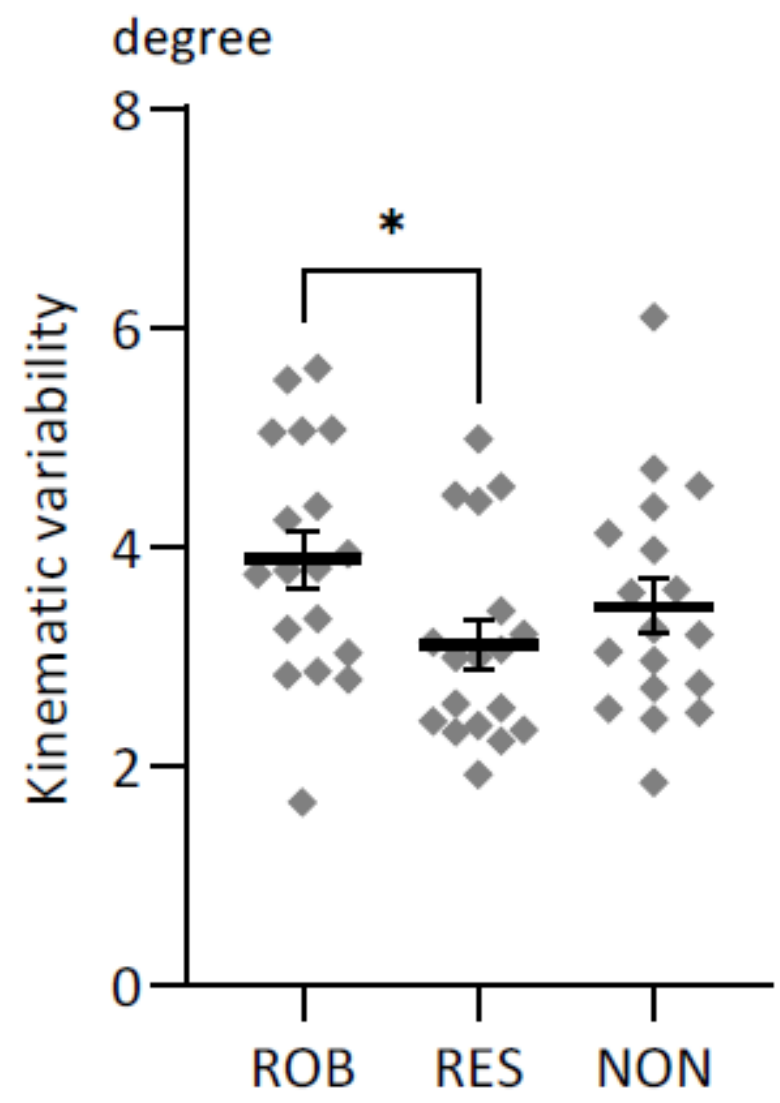

Figure 2

The average changes in kinematic variability for three conditions. Comparisons of average changes in kinematic variability for ROB, RES, and NON. ${ }^{*} p<0.05$. Data are expressed as the mean with standard error (SE). 
A
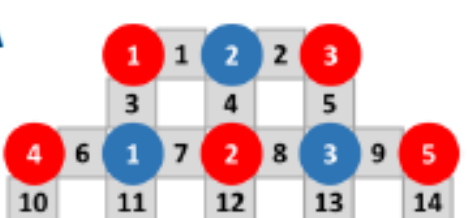

$\begin{array}{lllllllllll}4 & 15 & 6 & 16 & 7 & 17 & 8 & 18 & 5\end{array}$

\begin{tabular}{|l|l|l|l|l|}
19 & 20 & 21 & 22 & 23 \\
\hline
\end{tabular}

\begin{tabular}{lllllllllll}
14 & 24 & 6 & 25 & 7 & 26 & 8 & 27 & 15 \\
\hline
\end{tabular}

\begin{tabular}{ll|l}
28 & 29 & 30 \\
\hline
\end{tabular}

$\begin{array}{llllll}9 & 31 & 10 & 32 & 11\end{array}$

\begin{tabular}{l|l|l|}
33 & 34 & 35 \\
\hline
\end{tabular}

$\begin{array}{lllll}9 & 36 & 10 & 37 & 11\end{array}$

\begin{tabular}{l|l|l|}
38 & 39 & 40 \\
\hline
\end{tabular}

$\begin{array}{lllll}12 & 41 & 12 & 42 & 13\end{array}$

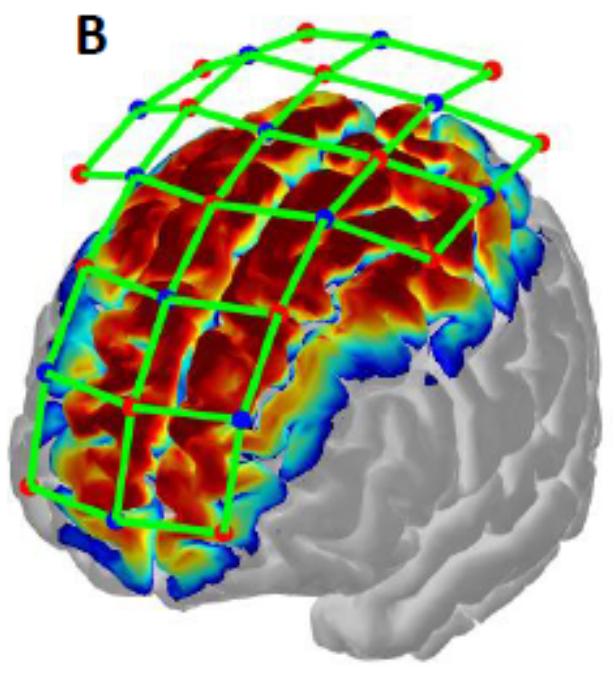

C

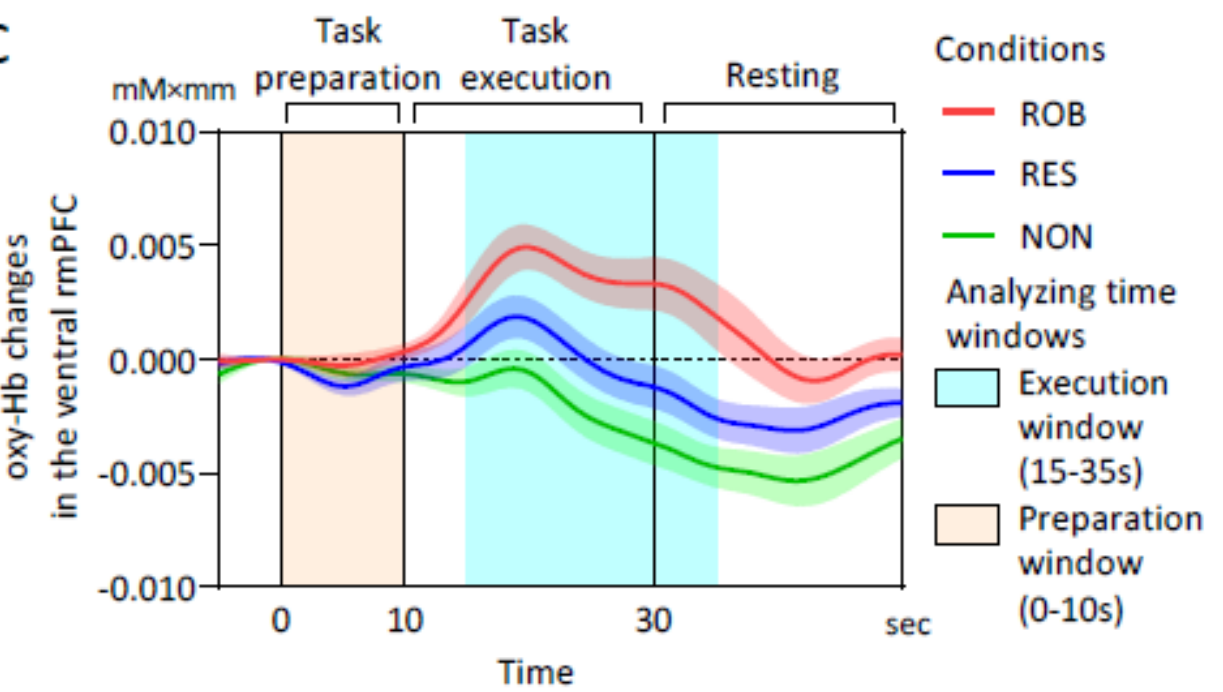

Figure 3

fNIRS data acquisition. (A) fNIRS optode layout design. Red and blue filled circles represent light sources and detectors, respectively. Yellow and grey rectangles represent long separation channels with and without short separation channels, respectively. The midline central point $(\mathrm{Cz})$ was located underneath the 12th channel. The source-detector distance was $3.0 \mathrm{~cm}$. (B) The cortical mapping shows estimated spatial information of the measurement on the surface of the cerebral cortex using the current fNIRS optode configuration. (C) Grand-average oxy-Hb responses in the ventral rmPFC during ROB (red line), RES (blue line), and NON (green line). The task onset was at 0 on the $x$-axis. The time-series were corrected to the baseline defined as the mean value over $2 \mathrm{~s}$ prior to the onset. fNIRS, functional nearinfrared spectroscopy; oxy-Hb, oxy-hemoglobin; rmPFC, rostromedial prefrontal cortex. Data are expressed as the mean with standard error (SE). 
A
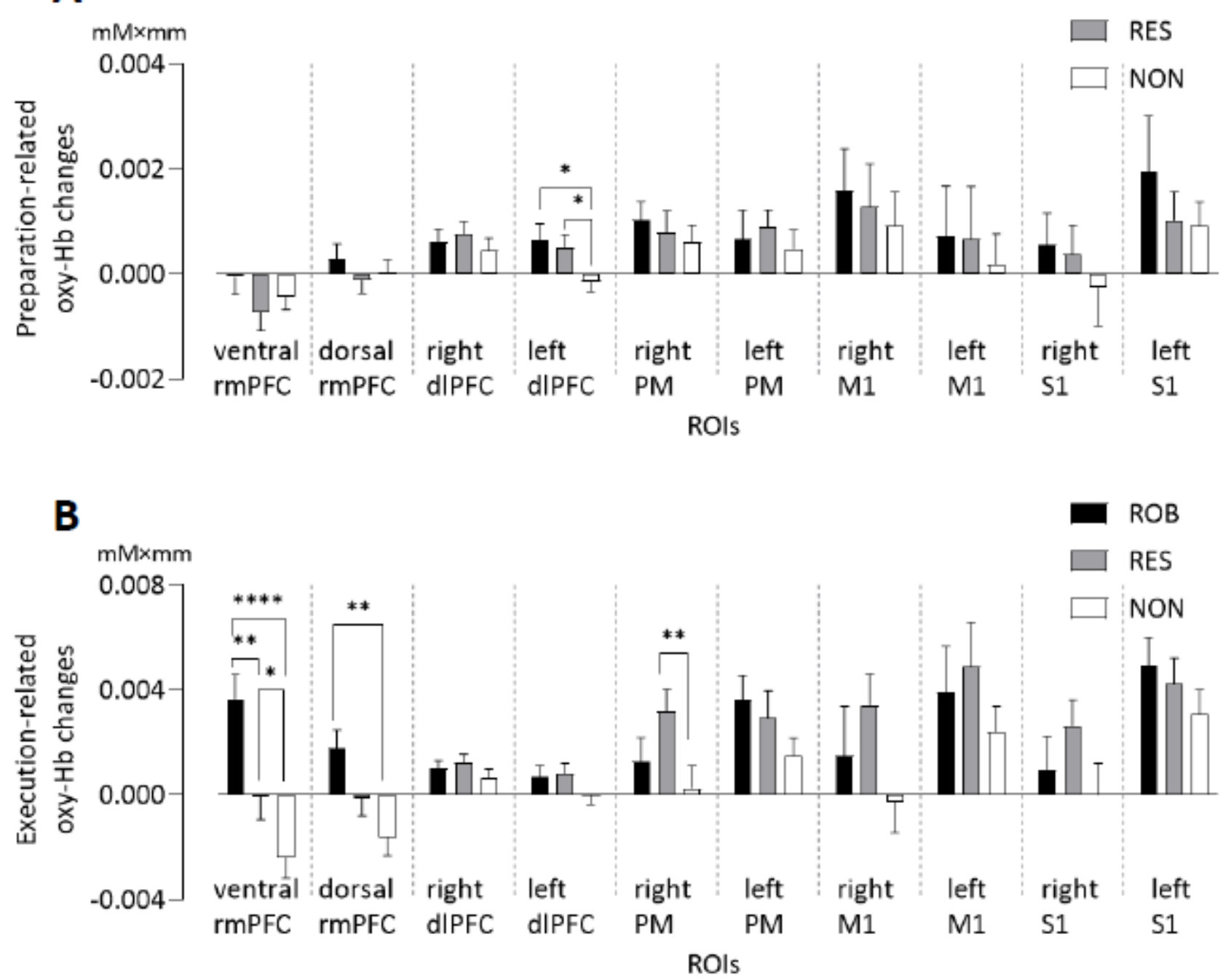

Figure 4

The average changes in oxy-Hb concentration of ROls for three conditions. Comparisons of $(A)$ preparation-related, and (B) execution-related oxy-Hb changes of ROls for ROB, RES, and NON. oxy-Hb, oxy-hemoglobin; ROIs, regions of interest; rmPFC, rostromedial prefrontal cortex; dIPFC, dorsolateral prefrontal cortex; PM, premotor area; M1, primary motor cortex; S1, primary somatosensory cortex. ${ }^{*} \mathrm{p}<$ $0.05,{ }^{*} \mathrm{p}<0.01,{ }^{\star \star \star \star} \mathrm{p}<0.0001$. Data are expressed as the mean with standard error (SE). 

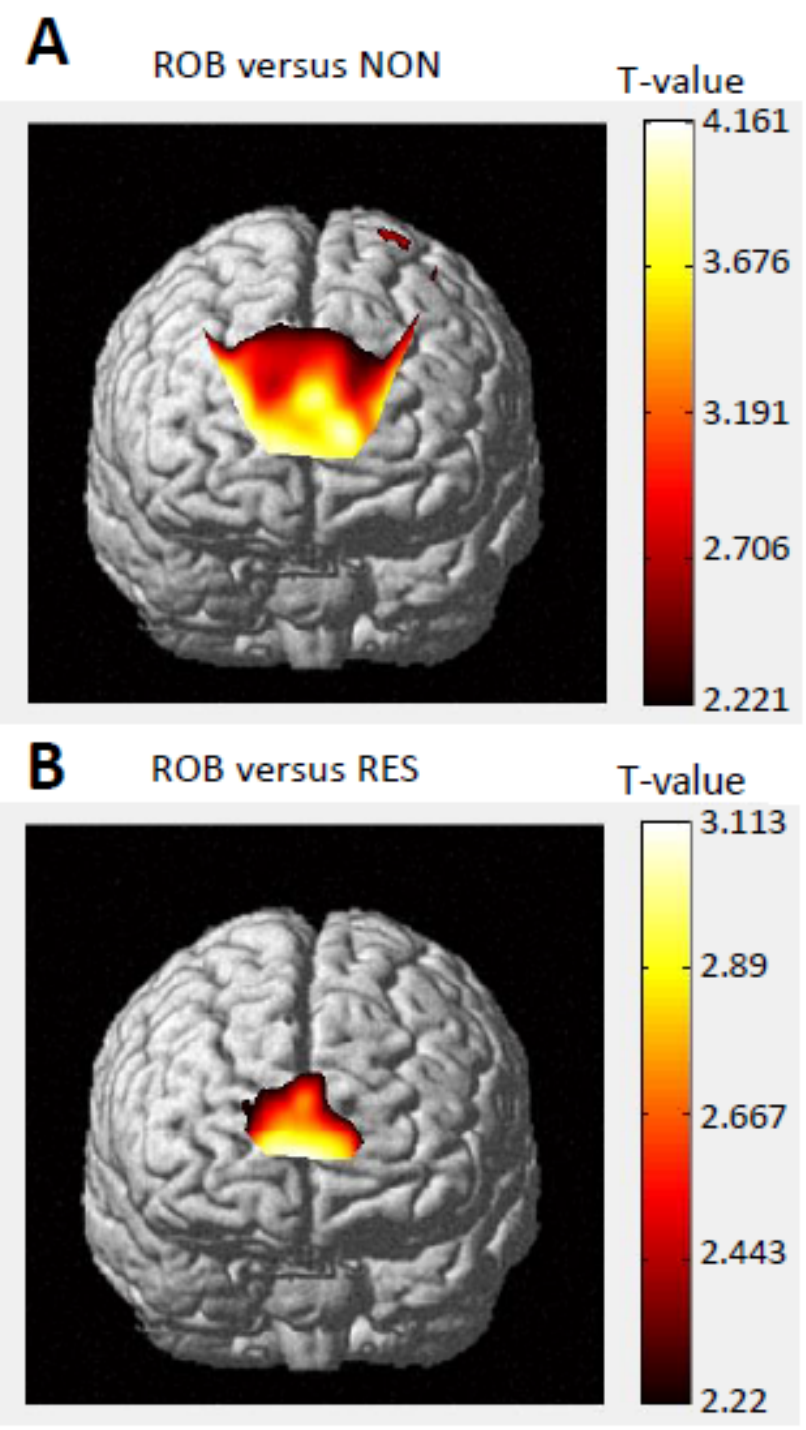

\section{Figure 5}

Group-level t-statistic map. Execution-related cortical activations, as determined by NIRS-SPM, for the comparisons between (A) ROB versus NON, and (B) ROB versus RES $(p<0.0167)$. Higher t-values represent relatively higher levels of cortical activation. 


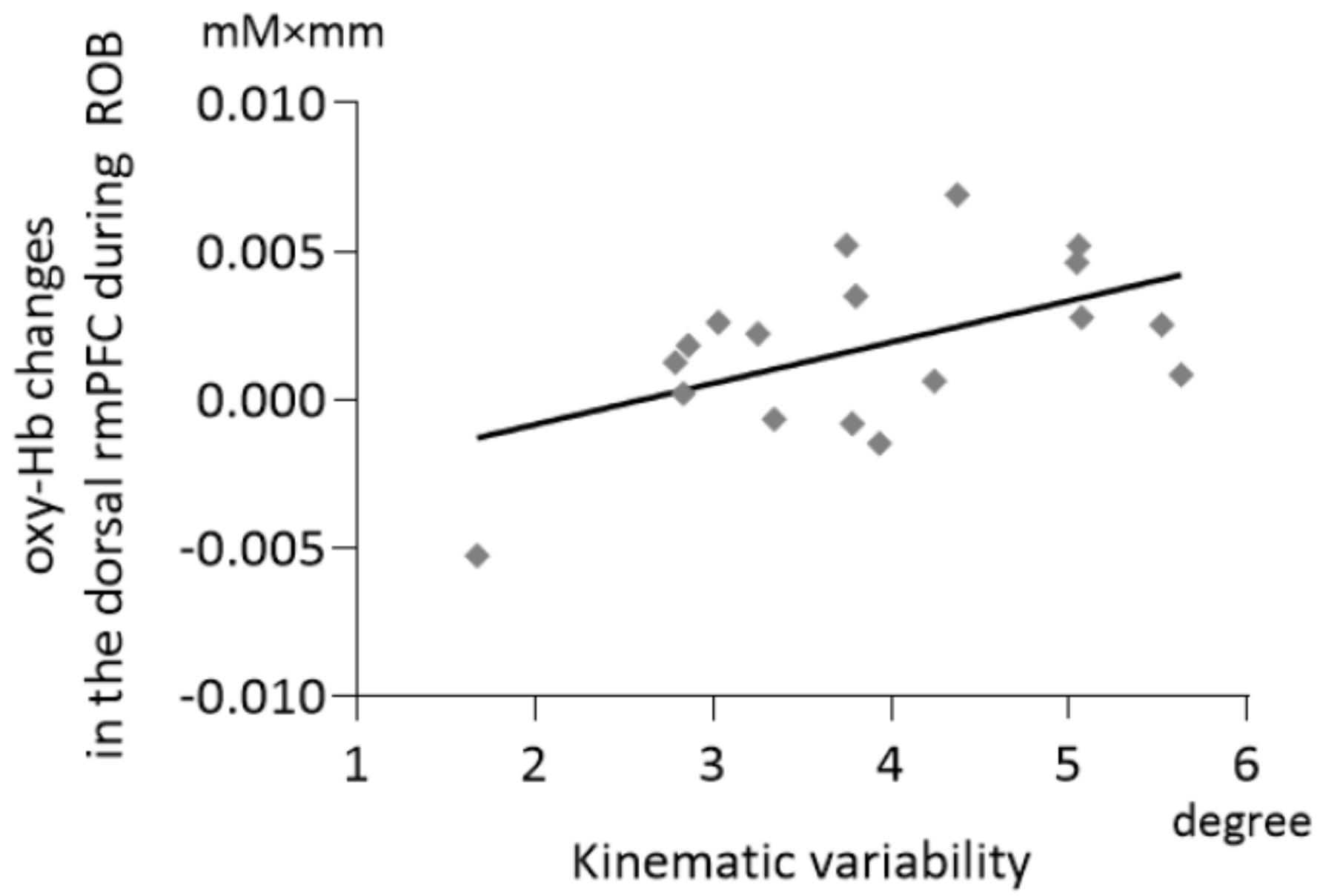

Figure 6

Correlation between oxy- $\mathrm{Hb}$ responses in the dorsal rmPFC during ROB and kinematic variability. The oxy$\mathrm{Hb}$ responses in the dorsal rmPFC during ROB were positively correlated with kinematic variability $(r=$ $0.523, p=0.026)$. oxy-Hb, oxy-hemoglobin; rmPFC, rostromedial prefrontal cortex. 